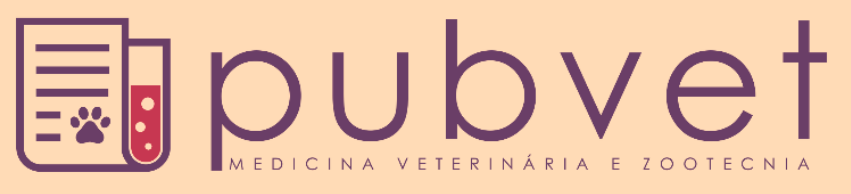

https://doi.org/10.31533/pubvet.v15n07a860.1-9

\title{
Dirofilariose ectópica em paciente canino assintomático: Relato de caso
}

\author{
Larisse Lustosa Pereira ${ }^{*} \bullet$, Ponan Mattoso Rubim², Nathália Maques de Oliveira Lemos ${ }^{3}$, Bruno \\ Ricardo Soares Alberigi $^{4} \bullet$ (D), Alexandre José Rodrigues Bendas ${ }^{4} \bullet$ (D), Mário dos Santos Filho ${ }^{\circ}$ (D) \\ ${ }^{I}$ Discente do curso de graduação em Medicina Veterinária, Universidade de Vassouras. Vassouras - RJ Brasil. \\ ${ }^{2}$ Médico Veterinário Autônomo, Rio de Janeiro - RJ Brasil. \\ ${ }^{3}$ Discente de Mestrado - Programa de pós-graduação em Medicina Veterinária, Universidade Federal Rural do Rio de Janeiro, Seropédica-RJ Brasil. \\ ${ }^{4}$ Docente do Departamento de Medicina e Cirurgia Veterinária, Universidade Federal Rural do rio de Janeiro, Seropédica-RJ Brasil. \\ ${ }^{5}$ Docente do curso de Graduação em Medicina Veterinária, Universidade de Vassouras. Vassouras - RJ Brasil. \\ *Autor para correspondência, E-mail: larilustosap@gmai.com
}

Resumo. A dirofilariose é uma doença parasitária decorrente da infecção provocada pelo nematódeo Dirofilaria immitis, transmitida por meio da inoculação de formas larvais de terceiro estágio, após picada de culicídeos vetores. É comum em cães e menos frequente em gatos, mas também afeta outros hospedeiros, como carnívoros selvagens e humanos. Sua distribuição tem importância de caráter mundial. No Brasil, a dirofilariose canina é endêmica e estima-se que a prevalência nacional esteja em torno de $23,1 \%$, com sua transmissão ocorrendo principalmente em cidades litorâneas e de clima quente, onde existe maior probabilidade do desenvolvimento dos vetores. A gravidade da doença pode ser decorrente da cronicidade dessa infecção e pela quantidade de parasitos no lúmen vascular, decorrendo em lesões em nível endotelial e formações de trombos. Quando o animal já se encontra em estágio mais avançado da doença, ele pode apresentar síndrome da veia cava, que é comumente fatal por conta do acúmulo de parasitos adultos que se deslocam das artérias pulmonares e invadem ventrículo e átrio direito, podendo chegar na desembocadura da veia cava, evoluindo desta forma, para um processo de congestão do sistema venoso. Nos casos de grandes infestações e nos casos em que o animal apresente intolerância a exercícios físicos, não se deve forçar atividade, pois isso pode estimular a translocação dos parasitas, levando a um processo obstrutivo embólico. Casos de ciclos erráticos são pouco reportados, e quando ocorrem, acometem animais com alta taxa de infestação. O presente trabalho tem por objetivo relatar um caso clínico de um paciente assintomático, com manifestação errática da doença, percebida em cavidade abdominal após a realização de procedimento cirúrgico de ovário-histerectomia. Foram realizados alguns exames, após a detecção do verme adulto no procedimento cirúrgico, para avaliar o estadiamento da doença. Contudo, os mesmos não apresentaram alterações significantes. Foi iniciado protocolo de tratamento, utilizando a doxiciclina como base para o controle da bactéria simbionte para o nematódeo, a Wolbachia spp. associada à lactona macrocíclica (moxidectina), como forma de controle da microfilaremia. $\mathrm{O}$ paciente continua em observação, sem manifestação de sinais clínicos.

Palavras chave: Assintomático, cão, ciclo errático, Dirofilaria immitis

\section{Ectopic Heartworm in an Asymptomatic Canine Patient: Case Report}

Abstract. Heartworm disease is a parasitic disease resulting from the infection caused by the nematode Dirofilaria immitis, transmitted through the inoculation of third stage larval forms, after stinging culicidae vectors. It is common in dogs and less frequent in cats, but it also affects other hosts, such as wild carnivores and humans. Its distribution is important worldwide. In Brazil, canine heartworm disease is endemic and it is estimated that the national prevalence is around $23.1 \%$, with its transmission occurring mainly in coastal 
cities and in hot climates, where there is a greater probability of the development of the vectors. The severity of the disease may be due to the chronicity of this infection and the number of parasites in the vascular lumen, resulting in lesions at the endothelial level and thrombus formations. When the animal is already in a more advanced stage of the disease, it can present vena cava syndrome, which is commonly fatal due to the accumulation of adult parasites that move from the pulmonary arteries and invade the right ventricle and atrium, which can reach the mouth of the vena cava, evolving in this way, to a process of congestion of the venous system. In cases of major infestations and in cases where the animal has intolerance to physical exercise, activity should not be forced, as this can stimulate the translocation of the parasites, leading to an embolic obstructive process. Cases of erratic cycles are rarely reported, and when they occur, they affect animals with a high rate of infestation. The present study aims to report a clinical case of an asymptomatic patient, with an erratic manifestation of the disease, perceived in the abdominal cavity after undergoing an ovariohysterectomy surgical procedure. Some tests were carried out, after detecting the adult worm in the surgical procedure, to assess the stage of the disease. However, they did not show significant changes. A treatment protocol was started, using doxycycline as a basis for the control of the symbiotic bacteria for the nematode, Wolbachia spp. associated with macrocyclic lactone (moxidectin), as a way of controlling microfilaremia. The patient remains under observation, with no clinical signs.

Keywords: Asymptomatic, dog, erratic cycle, Dirofilaria immitis

\section{Dirofilariasis ectópico en un paciente canino asintomático: Reporte de caso}

Resumen. La dirofilariasis es una enfermedad parasitaria resultante de la infección causada por el nematodo Dirofilaria immitis, transmitida por la inoculación de formas larvarias del tercer estadio, tras la picadura de los vectores culicidae. Es común en perros y menos frecuente en gatos, pero también afecta a otros huéspedes, como carnívoros salvajes y humanos. Su distribución es importante a nivel mundial. En Brasil, la dirofilariosis canina es endémica y se estima que la prevalencia nacional ronda el $23,1 \%$, con su transmisión ocurriendo principalmente en ciudades costeras y en climas cálidos, donde existe una mayor probabilidad de desarrollo de los vectores. La gravedad de la enfermedad puede deberse a la cronicidad de esta infección y al número de parásitos en la luz vascular, lo que da lugar a lesiones a nivel endotelial y formaciones de trombos. Cuando el animal ya se encuentra en una etapa más avanzada de la enfermedad, puede tener síndrome de la vena cava, que comúnmente es fatal debido a la acumulación de parásitos adultos que se mueven desde las arterias pulmonares e invaden el ventrículo y la aurícula derechos, que pueden llegar a la boca de la vena cava, evolucionando así, a un proceso de congestión del sistema venoso. En casos de infestaciones importantes y en los casos en que el animal tenga intolerancia al ejercicio físico, no se debe forzar la actividad, ya que esto puede estimular la translocación de los parásitos, dando lugar a un proceso embólico obstructivo. Rara vez se informan casos de ciclos erráticos y, cuando ocurren, afectan a animales con una alta tasa de infestación. El presente estudio tiene como objetivo reportar un caso clínico de una paciente asintomática, con una manifestación errática de la enfermedad, percibida en la cavidad abdominal luego de ser sometida a un procedimiento quirúrgico de ovariohisterectomía. Se realizaron algunas pruebas, luego de detectar el gusano adulto en el procedimiento quirúrgico, para evaluar el estadio de la enfermedad. Sin embargo, no mostraron cambios significativos. Se inició un protocolo de tratamiento, utilizando doxiciclina como base para el control de las bacterias simbióticas del nematodo Wolbachia spp. asociado con lactona macrocíclica (moxidectina), como una forma de controlar la microfilaremia. El paciente permanece en observación, sin signos clínicos.

Palabras clave: Asintomático, perro, ciclo errático, Gusano del corazón, Dirofilaria immitis 


\section{Introdução}

A dirofilariose trata-se de uma doença causada pelo nematódeo Dirofilaria immitis também conhecida como verme do coração (Silva \& Langoni, 2009). Sua transmissão ocorre por meio do mosquito da família Culicidae, que estejam parasitados com a larva da dirofilaria em seu estágio infectante-L3 (Romero-Rodríguez et al., 2019; Tzipory et al., 2010; Wang et al., 2016). Após o contágio, as microfilárias migram por meio do sistema circulatório, e se alojam principalmente nas artérias pulmonares e pulmões dos cães domésticos, sendo estes os hospedeiros definitivos. Dentre os sinais clínicos observados, a fraqueza e anorexia podem estar presentes, tempo de preenchimento capilar aumentado (Martins et al., 2019). A Dirofilaria immitis pode ser prevenida com a administração de lactonas macrocíclicas, necessitando negatividade do teste de detecção de microfilárias e detecção de antígeno para o verme adulto. Como forma de tratamento, esta base também está indicada, uma vez que em até 30 dias pode eliminar as larvas infectantes, sendo assim indicada a administração mensal para impedir a disseminação de larvas infectantes, que os mosquitos possam introduzir e/ou no repasto sanguíneo adquirir as formas larvais e picar um novo animal, persistindo o ciclo (ESDA, 2017). Ainda com relação ao tratamento, como a base adulticida não está disponível no Brasil, o uso da doxiciclina vem sendo utilizado, justamente por combater a bactéria simbionte do parasita, sendo feita, desta forma, o protocolo "slow-kill", onde com o tempo os parasitas adultos vão morrendo. De forma preventiva, é muito importante manter a orientação e a realização de testes em animais assintomáticos, independentemente do local de residência, e deve ser reforçada em áreas endêmicas (Trancoso et al., 2020). Tendo em vista algumas formas de contaminação atípica para a dirofilariose, o objetivo desse trabalho foi relatar um caso clínico de paciente assintomático, com manifestação errática da doença, percebida em cavidade abdominal após a realização durante procedimento cirúrgico de ováriohisterectomia.

\section{Relato de caso}

Foi atendido, em uma clínica veterinária do município do Rio de Janeiro, no bairro de Ilha de Guaratiba, um paciente canino, fêmea, sem raça definida, com aproximadamente dois anos de idade (Figura 1), para avaliação e realização do procedimento de castração. A paciente tinha hábito de vida peridomiciliado, sendo cuidada por moradores do bairro, e foi levada para realização do procedimento, por uma de suas cuidadoras, que mencionou que a cadela não apresentava alterações clínicas.

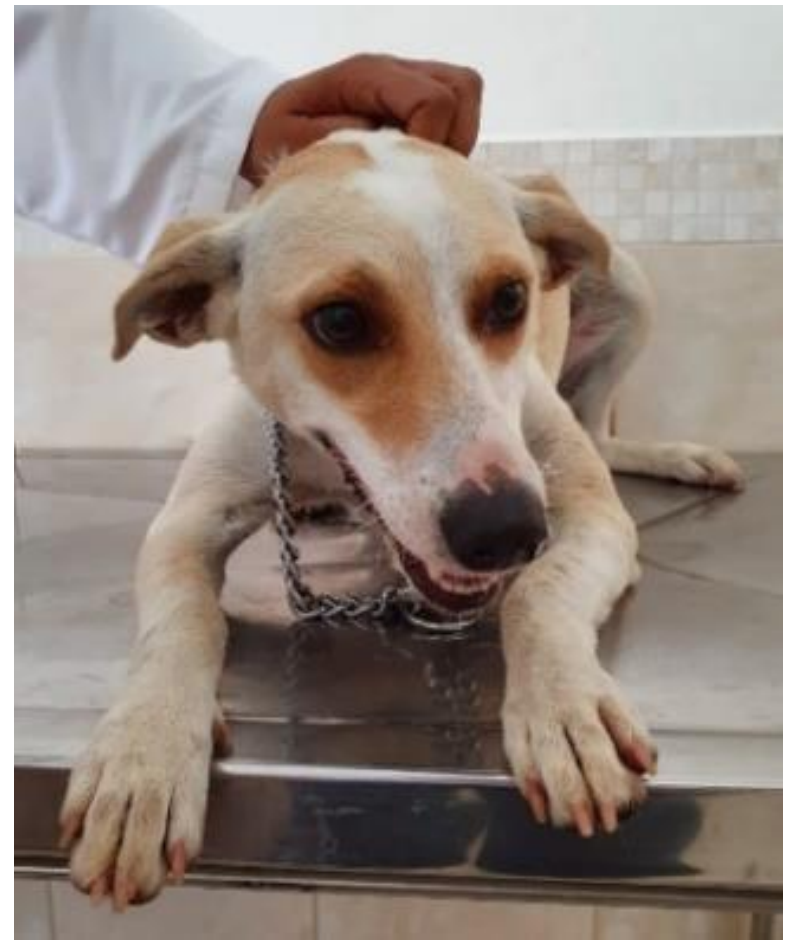

Figura 1. Paciente canina, fêmea, acometida por dirofilariose, assintomática, da descrição do presente relato de caso. 
Procedeu-se a avaliação física, estando os parâmetros vitais dentro da faixa de normalidade, tais quais: mucosas aparentes apresentavam-se normocoradas e auscultas cardíaca e respiratória sem alterações. Coletou-se amostras de sangue para avaliação do hemograma e bioquímica sérica hepática tais como Fosfatase alcalina, Aspartato amino transferase e Alanina amino transferase (FA, ALT e AST) e renal (ureia e creatinina). O hemograma apresentou, como única alteração, eosinofilia de 13\% (ref.: 2$10 \%$ ), enquanto a bioquímica apresentava-se sem alterações. Não foram observadas hematozoários na amostra coletada. Desta forma, procedeu-se a realização da cirurgia. Foi realizada ovário-histerectomia $(\mathrm{OSH})$, sem intercorrências no transoperatório. Contudo, durante a incisão, após a abertura na linha alba foi detectado um filarídeo dentro da cavidade abdominal (Figura 2). O mesmo foi retirado, em único exemplar, e visualizado que a porção terminal se espiralava. Logo após a recuperação do paciente, no pós-operatório imediato, foi solicitado pesquisa de antígeno de $D$. immitis, teste de Knott modificado, para pesquisa de microfilárias, radiografia torácica e ecodopplercardiograma, e passadas orientações à tutora quanto da realização destes, tão breve possível. Passados 10 dias, após a recuperação da paciente, e realizada a retirada de pontos cirúrgicos, foi realizado o teste Snap 4DX Plus®, onde confirmou-se a presença de antígenos de D. immitis. O teste de Knott revelou presença de microfilárias.

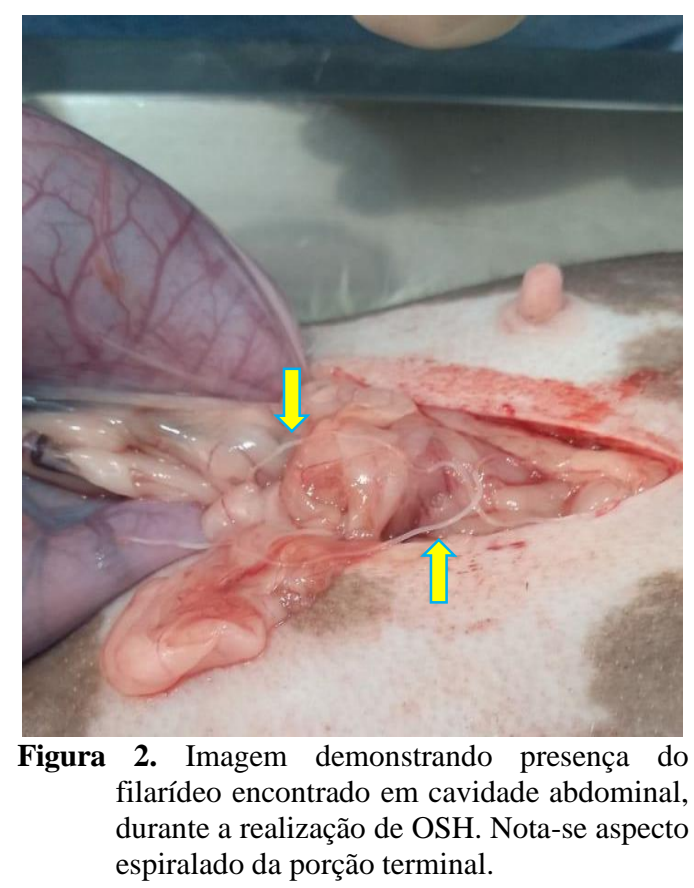

A imagem radiográfica demonstrou discreto padrão bronquial e intersticial em campos pulmonares, nas regiões dorso-caudais (Figura 3 e 4 ).

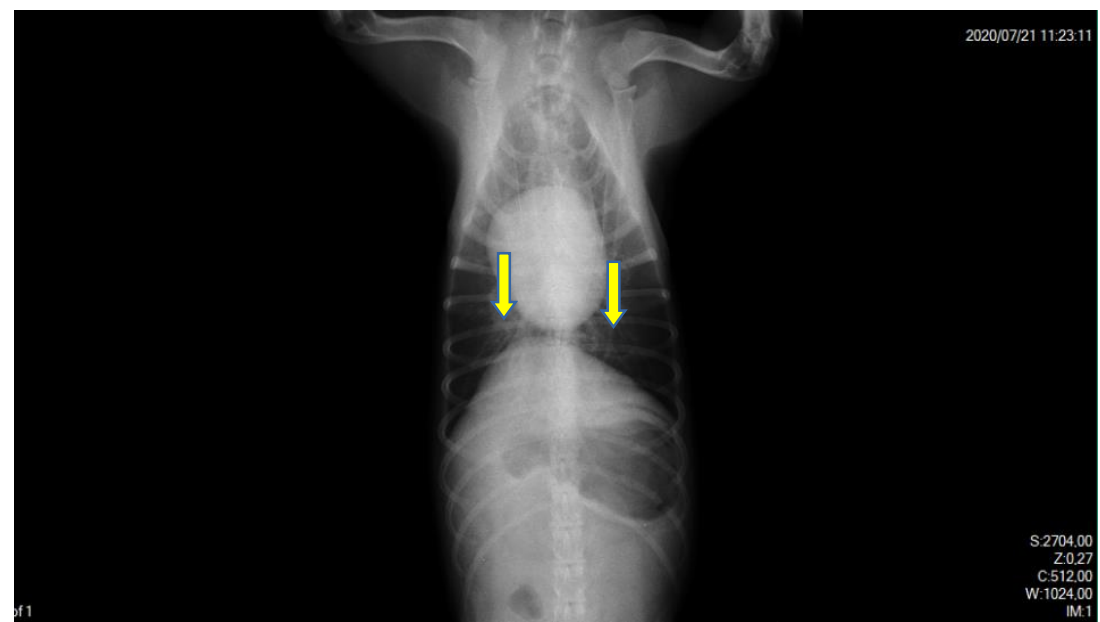

Figura 3. Imagem radiográfica da paciente, em projeção ventro-dorsal, revelando padrão bronquial e intersticial em campos pulmonares (pontas de setas). 


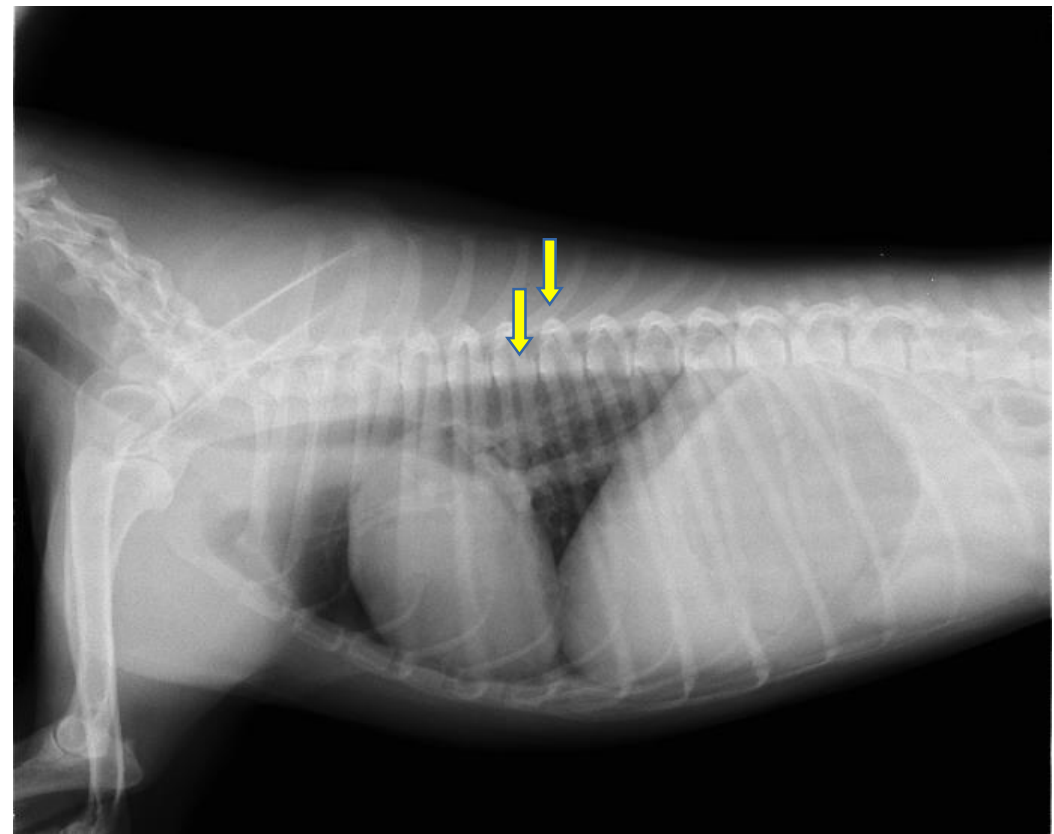

Figura 4. Imagem radiográfica da paciente, em projeção latero-lateral, revelando padrão bronquial e intersticial em campos pulmonares (pontas de setas).

Ao exame ecodopplercardiográfico, não foram visualizados parasitas nas artérias pulmonares ou cavidades cardíacas direitas, tendo como alteração discreta insuficiência valvar mitral, sem repercussão hemodinâmica (Figura 5 e $\underline{6}$ ).

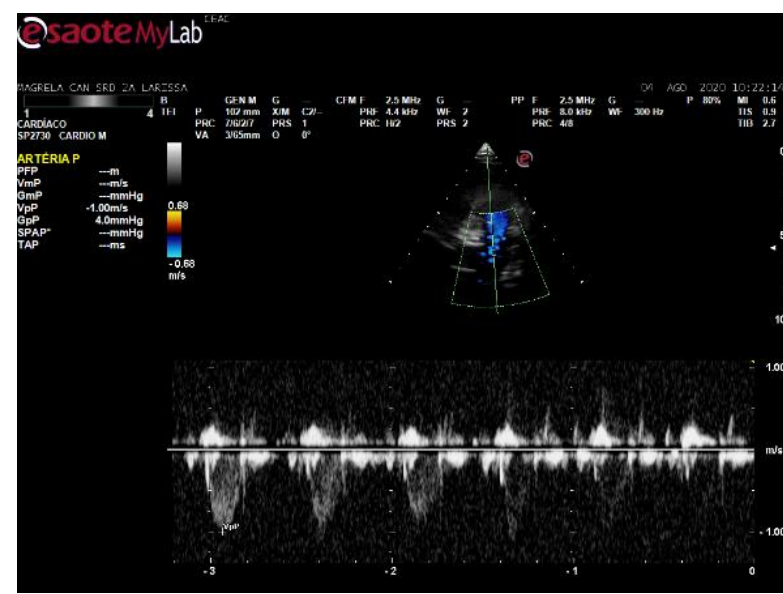

Figura 5. Exame ecodopplercardiográfico da paciente, em modo $\mathrm{M}$, evidenciando o fluxo da artéria pulmonar. Fonte: Bruno Alberigi, 2020.

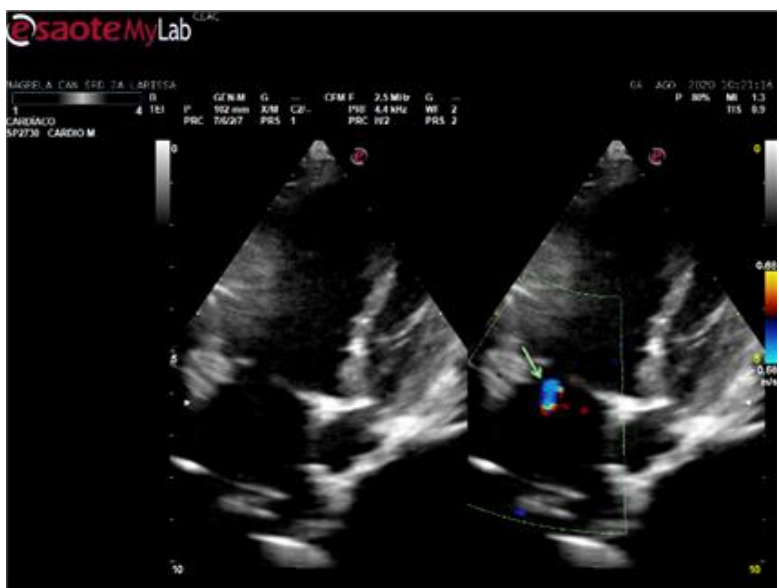

Figura 6. Exame ecodopplercardiográfico da paciente, em modo bidimensional, no corte apical quatro câmaras, evidenciando discreto refluxo de válvula mitral. Fonte: Bruno Alberigi, 2020.

Da mesma forma, o exame realizou a mensuração dos diâmetros de átrio esquerdo e da aorta, a fim de realizar a mensuração da relação, onde os valores foram $1,96 \mathrm{~cm}$ e 1,78 cm, respectivamente, com relação de 1,10 (Figura 7).

Ao exame ecodopplercardiográfico, não foram visualizados parasitas nas artérias pulmonares ou cavidades cardíacas direitas, tendo como alteração discreta insuficiência valvar mitral, sem repercussão hemodinâmica (Figura 5 e 6 ). Da mesma forma, o exame realizou a mensuração dos diâmetros de átrio esquerdo e da aorta, a fim de realizar a mensuração da relação, onde os valores foram 1,96 cm e 1,78 $\mathrm{cm}$, respectivamente, com relação de 1,10 (Figura 7).

Demais válvulas e morfologias estruturais de câmaras cardíacas, não apresentaram alterações, sendo confirmada discreta turbulência sistólica no interior do átrio esquerdo, pelo método Doppler, evidenciando a insuficiência mitral é de grau discreto. 
Após interpretação dos resultados dos exames, foi iniciado o tratamento via oral à base de omeprazol $1 \mathrm{mg} / \mathrm{kg}$, uma vez ao dia, em jejum por 30 dias, doxiciclina $10 \mathrm{mg} / \mathrm{kg}$, a cada $12 \mathrm{~h}$, durante 30 dias, prednisolona $1 \mathrm{mg} / \mathrm{kg}$ uma vez ao dia, durante cinco dias e, para uso externo, foi prescrito moxidectina $2,5 \%$ + imidacloprida $10 \%$ tópico (Advocate ${ }^{\circledR}$ cães) a cada 30 dias, para uso contínuo. O paciente se encontra no décimo mês de tratamento, sem apresentar efeitos colaterais em relação ao mesmo, se mantendo assintomático.

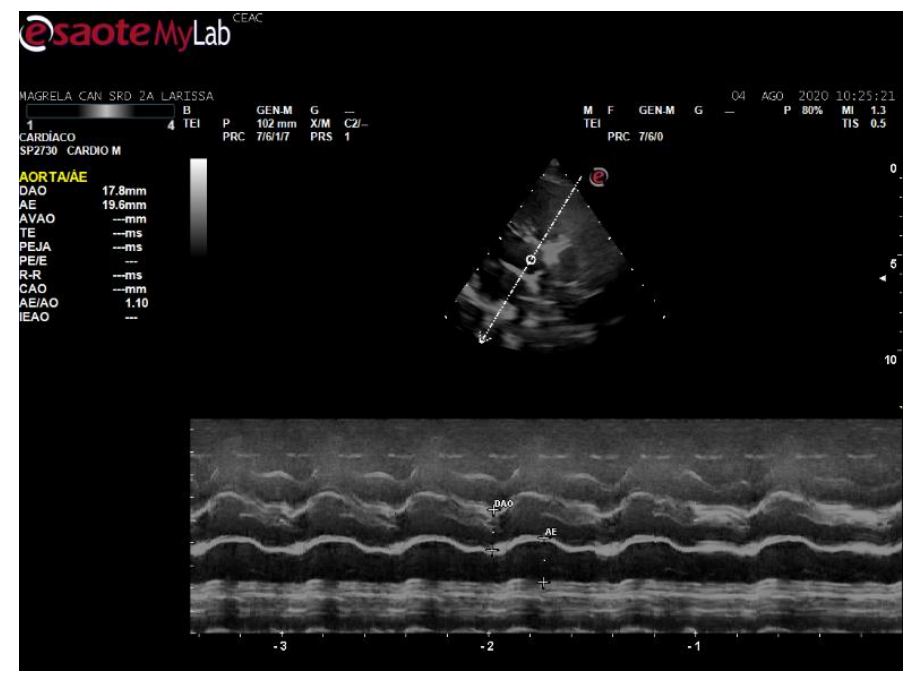

Figura 7. Exame ecodopplercardiográfico da paciente, em modo $\mathrm{M}$, no corto apical com via de saída de Ventrículo Esquerdo, evidenciando relação átrio esquerdo/aorta de 1,10. Fonte: Bruno Alberigi, 2020.

\section{Discussão}

O paciente vivia nas ruas, em área endêmica com o clima predominantemente quente e úmido e próximo à região litorânea, que são pontos importantes a serem considerados na epidemiologia da doença, principalmente com a probabilidade de infecção (Bendas et al., 2019; Labarthe \& Guerrero, 2005).

Antes da realização do procedimento cirúrgico, foi feito exame físico, e os parâmetros vitais estavam dentro da faixa de normalidade. Em muitos casos da doença o paciente pode ser assintomático como foi o que ocorreu nesse caso, por conta da idade da paciente, que tem ainda a seu favor o sistema imune (AHS, 2018). O curso assintomático da doença é frequentemente observado em cães parasitados, chegando a um percentual de $75 \%$ em alguns trabalhos (Meriem-Hind \& Mohamed, 2009).

Com relação à avaliação hematológica, a presença de eosinofilia é um importante achado para presença deste parasito (Moreira et al., 2019), uma vez que a infestação e lesão em nível vascular cursa com predomínio na mobilização dessas células, embora sem especificidade. Aliado a isto, a não detecção de hematozoários na amostra acaba por, nesse primeiro momento, não atentar para possível diagnóstico diferencial de dirofilariose (Silva \& Langoni, 2009).

Na realização do procedimento, a presença do filarídeo livre dentro da cavidade abdominal, por si, caracteriza sua migração errática, assim como Brener et al. (2012) também relatou que vermes adultos imaturos podem migrar erroneamente para locais incomuns como fígado, cérebro, espaço epidural, câmara anterior do olho, saco pericárdico, tecido subcutâneo, brônquios e cavidade peritoneal. Entretanto, este mesmo autor, não relata achados de verme livre em abdome. Esse achado gerou alerta, uma vez que duas situações podem ser conflitadas para a ocorrência do ciclo errático: uma migração totalmente aleatória do parasito ou uma carga parasitária extremamente alta (Nelson et al., 2020). Sendo a primeira a hipótese mais aceitável, uma vez que em cargas parasitárias altas provavelmente cursariam com achados clínicos importantes.

No momento da retirada do exemplar, foi possível perceber que na porção distal ocorreu um movimento do parasita de se espiralar. Esse comportamento é um achado característico de machos, 
e serve como diferenciação sexual (Romero-Rodríguez et al., 2019). Essa informação reflete diretamente na necessidade de confirmação do teste específico, uma vez que se a carga parasitária for baixa e na presença apenas de machos, pode haver pouca sensibilidade do teste (Alberigi et al., 2020).

Após a realização do procedimento cirúrgico, com posterior retorno da paciente para realização dos exames, foi realizado o exame específico para dirofilariose por meio do teste de imune ensaio rápido, assim como também foi utilizado por Labarthe et al. (2014) para comprovar que o animal era positivo, constatando-se a detecção do parasito, bem como a visualização de microfilárias de $D$. immitis no teste de Knott modificado. Isto denota que a não visualização de hematozoários na amostra, não necessariamente exclui o diagnóstico (Leite et al., 2012).

Ressalta-se que a detecção de presença de hematozoários, também não confirma ser de Dirofilaria immitis, ou de outros filarídeos, como as do gênero Acanthocheilonema sp. que possuem menor patogenicidade e pouca implicação clínica, fazendo-se necessário o uso do teste de concentração de Knott modificado, preconizado para a diferenciação e estadiamento, uma vez que remete a vermes adultos se reproduzindo (Trancoso et al., 2020).

Os achados de exames imaginológicos, não sugeriram alterações clássicas da doença, tais como padrão vascular em artérias pulmonares, na radiografia, e hipertensão pulmonar, ao ecodopplercardiograma (Martins et al., 2019), sendo mais um fator de reforçar e justificar o fato do animal apresentar-se assintomático. Contudo, revelou alguns achados incidentais, como o padrão bronquial e intersticial na imagem radiográfica, podendo se relacionar a processos alérgicos, tais como da própria presença do parasito, e discreto refluxo de mitral, sem traduzir importância clínica quando corroborado com os achados descritivos do ecodopplercardiograma, sem influenciar a hemodinâmica (Meireles et al., 2014). A não-observação de parasitas adultos nas artérias pulmonares trazem ainda uma confirmação plausível para a possibilidade do ciclo errático totalmente aleatório, e não necessariamente a infestação por uma alta carga parasitária, forçando os parasitas a migrarem para outros pontos (Nelson et al., 2020).

Diante da confirmação, o tratamento foi iniciado, fazendo-se uso de omeprazol, como forma de proteção da mucosa gástrica, antibiótico à base doxiciclina, preconizado para o método "slow-kill", sem ter grande impacto para possibilidade de eventos tromboembólicos (Alberigi et al., 2020). Ainda, o uso da lactona instituído, foi realizado com objetivo de controle da microfilaremia, impedindo o início de novos ciclos e a possibilidade de disseminação para outros animais, por meio do repasto sanguíneo do vetor (Labarthe et al., 2015). O uso da prednisolona reforça a importância de manter estável e permeável o sistema vascular, realizando a redução da inflamação decorrente do contato do parasita com as estruturas do sistema circulatório.

A estabilidade da paciente, frente aos achados clínicos normalizados, infere e reforça a importância e a natureza insidiosa da doença (Nelson et al., 2020), trazendo ainda mais atenção, e necessidade de testes diagnósticos para detecção de forma precoce da doença (Moraes et al., 2016).

\section{Conclusão}

As informações do relato, de forma invariavelmente epidemiológica, podem favorecer e reforçar a orientação de médicos veterinários, em relação à atenção a animais assintomáticos, que residem em regiões endêmicas. As medidas de orientação aos tutores locais quanto aos testes, e a instituição dos meios de profilaxia, de forma precoce, são medidas de grande impacto à saúde pública e animal.

\section{Referências}

American Heartworm Society. (2018). Current Canine Guidelines for the Prevention, Diagnosis, and Management of Heartworm Infection in Dogs.

Alberigi, B., Souza, C. da S. F., Fernandes, J. I., Merlo, A., \& Labarthe, N. (2020). Use of slow-release injectable moxidectin for treatment of Dirofilaria immitis infection during pregnancy. Frontiers in Veterinary Science, 6, 440. https://doi.org/10.3389/fvets.2019.00440.

Bendas, A. J. R., Branco, A. S., Silva, B. R. S. A., Paiva, J. P., Miranda, M. G. N., Mendes-de-Almeida, 
F., \& Labarthe, N. V. (2019). Mosquito abundance in a Dirofilaria immitis hotspot in the eastern state of Rio de Janeiro, Brazil. Veterinary Parasitology: Regional Studies and Reports, 18, 100320. https://doi.org/10.1016/j.vprsr.2019.100320.

Brener, B., Millar, P. R., Mattos, D. P. B. G., Uchôa, F., Bastos, B., Lyrio, I. R., Aragon, P. L., \& Sudré, A. P. (2012). Ectopic dirofilariosis in two dogs from Rio de Janeiro state, Brazil. Revista Do Instituto de Medicina Tropical de São Paulo, 54(3), 175-177. https://doi.org/10.1590/S003646652012000300011.

European Society of Dirofilariosis And Angiostrongylosis. (2017). Guidelines for clinical management of canine heartworm disease.

Labarthe, N., \& Guerrero, J. (2005). Epidemiology of heartworm: what is happening in South America and Mexico? Veterinary Parasitology, 133(2-3), 149-156. https://doi.org/10.1016/j.vetpar.2005.04.006.

Labarthe, N. V., Paiva, J. P., Reifur, L., Mendes-de-Almeida, F., Merlo, A., Pinto, C. J. C., Juliani, P. S., Almeida, M. A. O., \& Alves, L. C. (2014). Updated canine infection rates for Dirofilaria immitis in areas of Brazil previously identified as having a high incidence of heartworm-infected dogs. Parasites \& Vectors, 7(1), 1-8. https://doi.org/10.1186/s13071-014-0493-7.

Labarthe, N. V., Willi, L. M. V., Paiva, J. P., Miranda, M. G. N., Zoreck, K., \& Almeida, F. M. (2015). Chemoprophylaxis of Dirofilaria immitis (Leidy 1856) infection at a high challenge environment. Parasites \& Vectors, 8(1), 1-4. https://doi.org/10.1186 / s13071-015-1141-6.

Leite, L. C., Luz, E., Cirio, S. M., Leite, M. C., Molinari, H. P., Zadorosnei, A. C. B., Oliveira, T. M. F. S., \& Leite, S. C. (2012). Infecção de cães domésticos (Canis lupus familiaris, Linnaeus, 1758) da região metropolitana de Guaratuba, Paraná, Brasil por microfilárias circulantes de Acanthocheilonema reconditum (Grassi, 1899). Semina: Ciências Agrárias, 33(3), 1149-1155. https://doi.org/10.5433/1679-0359.2012v33n3p1149.

Martins, A. J. A., Sousa, V. C. S., \& Braga, Í. A. (2019). Dirofilariose canina: relato de caso. IV Colóquio Estadual de Pesquisa Multidisciplinar II Congresso Nacional de Pesquisa Multidisciplinar. Ciência e Tecnologia Em Busca de Inovações Empreendedoras.

Meireles, J., Paulos, F., \& Serrão, I. (2014). Dirofilariose canina e felina. Revista Portuguesa de Ciências Veterinárias, 109, 70-78.

Meriem-Hind, B.-M., \& Mohamed, M. (2009). Prevalence of canine Dirofilaria immitis infection in the city of Algiers, Algeria. African Journal of Agricultural Research, 4(10), 1097-1100. https://doi.org/10.5897/AJAR.9000561.

Moraes, M. F. C. V., Mendes-de-Almeida, F., Abdalla, L., Merlo, A., Paiva, J. P., \& Labarthe, N. V. (2016). Selamectin for the prevention of canine Dirofilaria immitis infection: field efficacy in clientowned dogs in a high risk area. Parasites \& Vectors, 9(1), 1-5. https://doi.org/10.1186/s13071-0161697-9.

Moreira, H. R., Madeira, E. A. O., Cunha, D. N. L., Scofield, A., Góes-Cavalcante, G., Abel, I., Guimarães, R. J. P. S., \& Fernandes, J. I. (2019). Dirofilaria immitis infection in dogs in Algodoal Island, Brazilian Amazon. Pesquisa Veterinária Brasileira, 39(7), 510-515. https://doi.org/10.1590/1678-6160-PVB-5916.

Nelson, T., McCall, J. W., \& Carinthers, D. (2020). Orientações atuais para prevenção, diagnóstico e controle da dirofilariose (Dirofilaria immitis) em cães. In American Hearhworm Society (pp. 1-6).

Romero-Rodríguez, P., García-y-González, E., Santos-Sotomaior, C., Pineda-Burgos, B., OlivarValladolid, G., Hernández-Ruiz, P., \& Ponce-Covarrubias, J. (2019). Prevalence of Dirofilaria immitis in domestic canines from two municipalities in the tropic of Guerrero, Mexico. Abanico Veterinario, 9(1), 1-11.

Silva, R. C., \& Langoni, H. (2009). Dirofilariose: zoonose emergente negligenciada. Ciência Rural, 39(5), 1615-1624. https://doi.org/10.1590/s0103-84782009005000062.

Trancoso, T. A. L., Lima, N. da C., Barbosa, A. S., Leles, D., Fonseca, A. B. M., Labarthe, N. V., Bastos, O. M. P., \& Uchôa, C. M. A. (2020). Detection of Dirofilaria immitis using microscopic, serological and molecular techniques among dogs in Cabo Frio, RJ, Brazil. Revista Brasileira de Parasitologia 
Veterinária, 29(1), 1-12. https://doi.org/10.1590/s1984-29612020009.

Tzipory, N., Crawford, P. C., \& Levy, J. K. (2010). Prevalence of Dirofilaria immitis, Ehrlichia canis, and Borrelia burgdorferi in pet dogs, racing greyhounds, and shelter dogs in Florida. Veterinary Parasitology, 171(1-2), 136-139.

Wang, S., Zhang, N., Zhang, Z., Wang, D., Yao, Z., Zhang, H., Ma, J., Zheng, B., Ren, H., \& Liu, S. (2016). Prevalence of Dirofilaria immitis infection in dogs in Henan province, central China. Parasite, 23(1-5). https://doi.org/10.1051/parasite/2016054.

Histórico do artigo:

Recebido: 26 de janeiro de 2021

Aprovado: 6 de março de 2021.
Licenciamento: Este artigo é publicado na modalidade Acesso Aberto sob a licença Creative Commons Atribuição 4.0 (CC-BY 4.0), a qual permite uso irrestrito, distribuição, reprodução em qualquer meio, desde que o autor e a fonte sejam devidamente creditados. 